\title{
Measles elimination and immunisation: national surveillance trends in Japan, 2008-2015
}

\author{
S. INAIDA ${ }^{1,2}$, S. MATSUNO ${ }^{3}$ AND F. KOBUNE \\ ${ }^{1}$ Department of Pharmacoepidemiology, Graduate School of Medicine and Public Health, Kyoto University, \\ Kyoto, Japan \\ ${ }^{2}$ Department of Epidemiology, Graduate School of Medicine, Dentistry and Pharmaceutical Sciences, Okayama \\ University, Okayama, Japan \\ ${ }^{3}$ Department of Architecture, Faculty of Architecture, Kogakuin University, Tokyo, Japan \\ ${ }^{4}$ National Institute of Infectious Diseases, Tokyo, Japan
}

Received 7 September 2016; Final revision 27 February 2017; Accepted 31 May 2017; first published online 23 June 2017

\section{SUMMARY}

Measles elimination relies on vaccination programmes. In Japan, a major outbreak started in 2007. In response, 5-year two-dose catch-up vaccination programme was initiated in April 2008 for children 13-16-years-old. In this study, we analysed the epidemic curves, incidence rates for each age group, virus genotype, vaccination coverage and ratio of measles gelatin particle agglutination (PA) antibody using surveillance data for 2008-2015.

Monthly case counts markedly decreased as vaccination coverage increased. D5, which is the endemic virus type, disappeared after 2011, with the following epidemic caused by imported viruses. Most cases were confirmed to have a no-dose or single-dose vaccination status. Although the incidence rate among all age groups $\geqslant 5$-years-old decreased during the study period, for children $<5$-years-old, the incidence rate remained relatively high and increased in 2014 . The ratio of PA antibody ( $\geqslant 1: 128$ titres) increased for the majority of age groups, but with a decrease for specific age groups: the $0-5$ months and the 2-4, 14, 19 and most of the 26-55- and the 60-year-old groups ( -1 to $-9 \%)$. This seems to be the result of higher vaccination coverage, which would result in decreasing natural immunity booster along with decreasing passive immunity in infants whose mothers did not have the natural immunity booster. The 20-29- and 30-39-year-old age groups had higher number of cases, suggesting that vaccination within these age groups might be important for eliminating imported viruses.

Key words: Antibody, epidemic, measles, national surveillance, vaccine.

\section{INTRODUCTION}

Measles is a highly contagious virus with direct contact or airborne transmission [1]. The severity of

\footnotetext{
* Author for correspondence: S. Inaida, Department of Pharmacoepidemiology, Graduate School of Medicine and Public Health, Kyoto University, Yoshidakonoe-cho, Sakyo-ku, Kyoto 606-8501, Japan.

(Email: inaida.shinako.7v@kyoto-u.ac.jp)
}

measles includes complications associated with other infectious diseases, such as pneumonia, encephalomyelitis (1/1000 cases) and other neurological abnormalities. In 2008, 164,000 measles-related deaths were reported worldwide [2]. The incidence of modified measles, which has milder symptoms owing to incomplete immunity and which can also arise from singledose vaccines [3], has increased, but is more difficult to diagnose than measles. Laboratory confirmation is 
recommended for $>80 \%$ of suspected cases in order to accurately detect the incidence rate of measles [4].

Although measles eradication could be accomplished by raising the population's immunity through vaccination programmes, it is difficult to maintain an appropriate ratio of vaccination coverage among large populations [5]. The efficacy rate of one-dose vaccination is only $90-95 \%$, and maintenance of two-dose vaccination coverage for $>95 \%$ of the entire population is recommended to prevent a measles epidemic [6]. The World Health Organization (WHO) announced the goal of achieving measles elimination in a WHO Western Pacific Region (WPRO) ( $<1$ case per million people) by 2012 [7]. They added Brunei, Darussalam, Cambodia and Japan to the list of countries from the WPRO from which measles had been eliminated as of 27 March 2015 [8]. However, many countries still have high measles incidence rates and very little vaccination coverage. Therefore, a worldwide elimination threshold has yet to be reached.

In Japan, measles epidemics have largely decreased after routine vaccination was initiated in 1978 [9, 10], with the total 10 -year number of deaths from measles having decreased from 2932 in the 1970s to 575 in the 1980s and 225 in the 1990s [11]. One-year-old children were primarily affected during those periods, and most of the epidemic peaks occurred in May. Rigorous measles vaccination programmes, using two doses of the vaccination, were not implemented until 2006, which nearly coincided with a major outbreak in 2007, during which an estimated 18000 children $<15$ years of age were affected [12]. A 5-year second-dose catch-up vaccination programme was initiated for children 13-16-years-old in April 2008, while a two-dose immunization schedule for children 1- and 5-6-years-old has been the national policy since 2006 [5]. National surveillance of measles cases has been strengthened since 2008 to include monitoring of all nationwide cases, virus genotyping and vaccination status. This replaced the previous, longstanding, sentinel surveillance system [5, 13]. The challenges for measles eradication are to administer the two-dose vaccine in a timely manner, maintain a vaccination coverage $>95 \%$ and continuously monitor incidence and susceptibility by confirming the measles-specific antibody seroprevalence among cohorts $[6,14,15]$. In the present study, we examined measles epidemics and the effects of the latest vaccination catch-up programme, using enhanced national surveillance data.

\section{MATERIALS AND METHODS}

\section{National surveillance data}

In this report, we describe aspects of national measles surveillance data from 2008 to 2015 . Differences in monthly case counts were examined using the Friedman test, a non-parametric test comparing observed datasets over time; with a $P$-value $<0 \cdot 01$ considered as significant. The null hypothesis stated that the sampled data would be identical among the different time points of data collection [16]. Statistical analysis was conducted using SPSS (ver. 21, IBM Corporation, USA). We created epidemic curves, calculated incidence rates per million by age group $(<1,1-4,5-9,10-14,15-19,20-29,30-39,40-49$ and $\geqslant 50$ years of age) and compared incidence rates from 2008 to 2014 . We summarized the vaccination coverage rates for this time period and described other key surveillance data, including country of origin of imported cases and antibody titre ratios.

We used data from the National Epidemiological Surveillance of Infectious Diseases (NESID) [9, 10], which included weekly counts of measles cases in Japan. The criteria for the reporting of measles cases were the presence of a generalized rash, fever $\left(101.3^{\circ} \mathrm{F}\right.$ or $\left.38.5^{\circ} \mathrm{C}\right)$, cough, coryza, or conjunctivitis. Commercial laboratory case confirmation, through the detection of measles-specific immunoglobulin $\mathrm{M}$ antibodies, was requested for all suspected cases.

Under Japan's Infectious Disease Control Law, physicians have been required to report all cases of defined notifiable diseases, including measles, since 2008. When clinicians encounter a measles case, they must report it to their public health centre immediately by phone or facsimile, even on holidays and weekends. The public health centre, in turn, must report it immediately to the prefectural government's Infectious Disease Surveillance Centre. The prefectural government enters the number of cases into the online NESID system. Then, the National Institute of Infectious Diseases (NIID) collects the information, including the name and mailing address of the hospital or clinic in which the patient was diagnosed, as well as the patient's age, sex and vaccination status (none, one or two doses), which were originally provided to the hospital on a patient- or parent-report basis. The vaccination coverage status (none, one or two doses) and the measles gelatin PA antibody for each age group were monitored from randomly selected healthy outpatients (with no chronic disease) who visited designated hospitals between July and 
September, each year. About 20 of the 47 prefectures in Japan were randomly selected by the Ministry of Health, Labour and Welfare for this random testing of gelatin PA. Results were reported to the NIID. The designated hospitals collected the vaccination status of patients through questionnaire on a self- or parent-report basis. The designated hospitals also collected the serological specimens for testing of measles PA antibody. Therefore, designated hospitals were randomly selected by the prefectural governments among the hospitals, which had the capacity to collect serological specimens for testing the measles PA antibody, as well as conduct the questionnaires for vaccine status. The targeted minimum number of specimens for PA antibody testing, along with vaccination status questionnaires, for each of the designated prefectures was 25 for each of the following age groups: $0-5$ months, 6-11 months, 1-69 years and $>70$ years. These serological specimens were collected with the informed consent or parental assent, as age appropriate and sent to the prefectural public health institutes that tested for the measles PA antibody.

Virus isolation and genotyping were conducted at prefectural public health institutes for all suspected cases of measles reported by a hospital or clinical, using reverse transcription polymerase chain reaction (RT-PCR) testing of samples and the results reported to the NIID [9]. A more detailed flow of our national surveillance programme has previously been described [17]. The surveillance data were based on the number of reported cases from hospitals, and no ethical consideration beyond obtaining informed consent [18].

\section{Incidence data analysis}

We calculated the incidence rates using 2010 national census data obtained from an online source run by the Japanese government [19] and compared the rates before the changes to the vaccination programme to those after the programmatic changes were implemented. We also attained the annual distribution of virus genotypes (2010-2015) and the annual average vaccination coverage from national data, as well as the proportion of reported vaccinated cases (20102014) [9]. These data were available in the NIID's Infectious Agents Surveillance Report (IASR) [9]. The IASR provided reports of the suspected countries in which the cases were infected, which were originally collected from randomly selected cases in an active surveillance programme.

\section{Changes in measles PA antibody}

We obtained the ratio of measles PA antibody ( $\geqslant 1: 16$ and $\geqslant 1: 128$ titres) in 2006 (before catch-up vaccinations were implemented) and for comparison with the ratio obtained for 2012-2015 after implementation of the programme. Positive measles antibody was defined as a PA antibody titre $\geqslant 1: 16$, with a titre $\geqslant 1: 128$ considered to be sufficient for immunity [9].

\section{RESULTS}

\section{Epidemic curve before and after the catch-up vaccination}

The Friedman test revealed a significant difference in the monthly number of cases $(P<0 \cdot 01)$ in all observed years. About 1300-2000 cases per month were identified in Japan between January and May 2008, when the catch-up vaccination programme was being implemented (Fig. 1). The number of cases of measles then decreased dramatically, with 11000 cases identified overall in 2008 and subsequently 200-700 cases between 2009 and 2014 (Supplementary Fig. S1). In 2015, only 36 cases were detected. No major peak outbreak, defined by $>100$ cases, was detected after 2008, except for a discrete peak of about 100 cases in May of 2009 and 2011. In 2008, 38\% of all the cases of measles reported were confirmed by laboratory testing, with this proportion increasing after the increase in vaccination rate in 2008, as follows: $72 \%$ in 2010 and 2011; $74 \%$ in 2012 and 2013; 92\% in 2014; and 91\% in 2015 .

The number of measles cases largely decreased for all age groups after the expansion of the vaccination programme in 2008 (Supplementary Figure S2). However, the number of cases remained relatively high for the 1-4-, 20-29- and 30-39-year-old age groups. Note that 2015 was not included in our analysis as only 36 cases of measles were detected, overall, that year. Between 2013 and 2014, the number of cases increased for most of age groups except for the $>40$-year-old age group. The proportion of age-based case was relatively high for the 15-19 (0.26) and 20-29 $(0 \cdot 22)$ years age groups in 2008 (Fig. 2). Between 2009 and 2014 , a relatively high proportion of cases $(0 \cdot 19$ $0 \cdot 21$ ) was identified for the $1-4,20-29$ and 30-39 years age groups.

The incidence rate per million people decreased remarkably across all age groups after the expansion of the vaccination programme in 2008 (Fig. 3). Again, note that 2015 was not included as only 36 cases were detected that year. Beginning in 2009, 


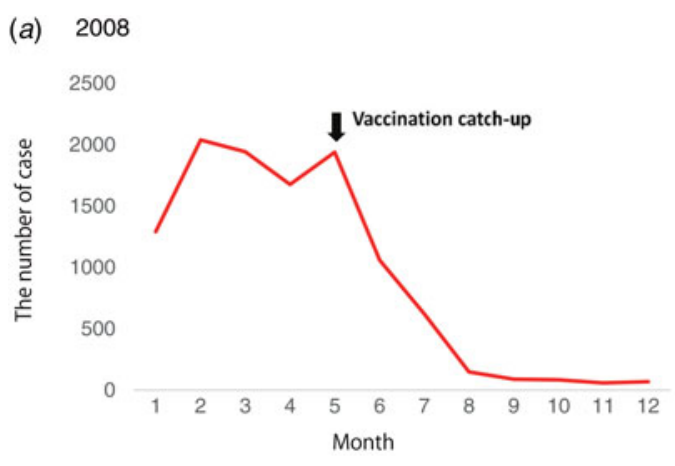

(b) 2009 to 2014

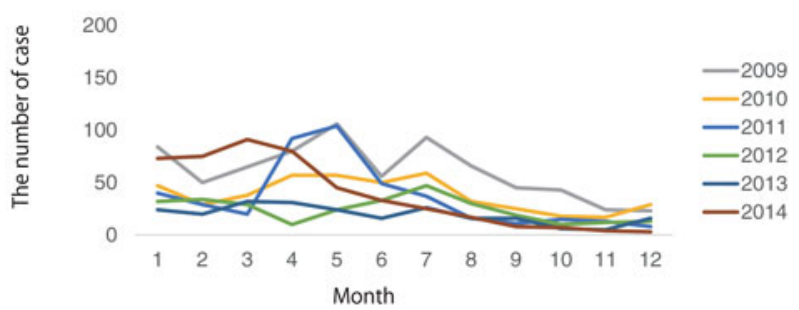

Fig. 1. Epidemic curve of measles case in Japan (20082014). The monthly number of measles cases was determined using data from the National Epidemiological Surveillance of Infectious Diseases (2015 was not included as only 36 cases were detected; The number of cases for (a) 2008 and (b) 2009-2014 is shown.

although a low incidence rate $(\leqslant 10$ cases per million people) was maintained among all age groups $\geqslant 5$ years, the incidence rate remained relatively high for the $<1$ - and 1-4-year-old age groups, with a sharp increase in 2014 , with $41 \cdot 9$ and $19 \cdot 3$ cases per million people, respectively.

\section{Vaccination coverage}

Vaccination coverage data were available for 6926 (2006), 7987 (2012), 8053 (2013) and 8620 (2014) individuals. The overall vaccination coverage rate for the first dose in 2006 was $>95 \%$, overall, for the 524-year-old cohort (excluding the approximate $50 \%$ of individuals with unknown vaccination status). However, a lower vaccination coverage rate was identified for the 15-, 16- and 17-year-old groups, who were also targeted for the 5-year catch-up vaccination programme implemented in 2008. In 2014, the coverage rate was also $>95 \%$, with a lower coverage rate of $92-93 \%$ for the 16,19 and $22-24$ age groups. The vaccination coverage rate for the second dose increased from $<10 \%$ in 2006 (among the 5-24-year-old cohort)

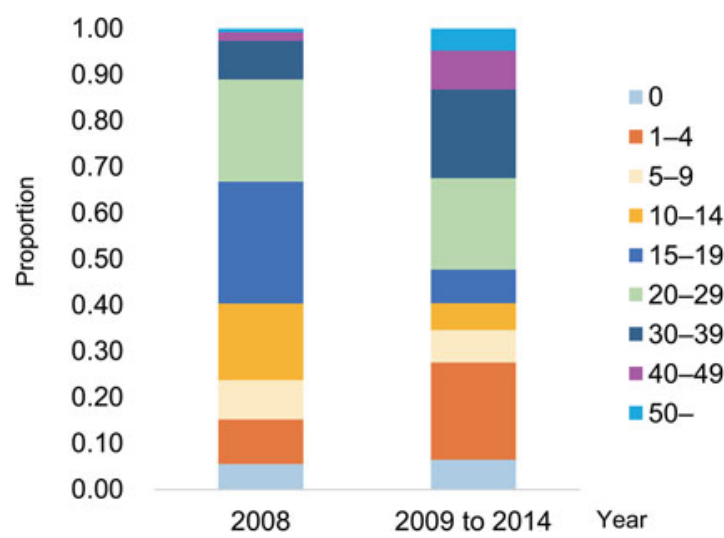

Fig. 2. Proportion of cases by age cohorts. The proportion of cases per age cohort was compared before and after the catch-up vaccination programme was implemented (2008, and the average between 2009 and 2014). Again, 2015 was not included as only 36 cases were detected.

to $50 \%$ in 2014 , although the target $95 \%$ coverage rate was not attained.

Among reported cases of measles between 2010 and 2014 , most of these individuals had received either no dose $(29 \cdot 7 \%)$ or one dose $(27 \cdot 8 \%)$ of the measlescontaining vaccine. A small percentage had received two doses of the measles-containing vaccine $(8 \cdot 8 \%)$. The vaccination status was unclear for $33.7 \%$ of reported cases of measles.

\section{Measles PA antibody}

The number of specimens available for PA antibody testing per year was as follows: 5992 (2006), 6860 (2012), 6980 (2013), 6786 (2014) and 6601 (2015). In 2006, a PA antibody titre $\geqslant 1: 16$ was obtained in $\geqslant 95 \%$ of all age cohorts $>2$ years, except for the $7-$, 9-, 10-, 15- and 17-year-old age groups. These age groups were targeted for the 5-year catch-up vaccination programme implemented in 2008. Over a 4-year period starting in 2012, a titre $\geqslant 1: 16$ was achieved in $\geqslant 95 \%$ of all age cohorts $>2$-years-old, with the exception of individuals in the $>70$-year-old cohort. In $2006, \geqslant 80 \%$ of all cohorts $>2$-years-old had PA antibody titres $\geqslant 1: 128$, except for the 10-, 14-, 15- and 21-year-old cohorts. Over the 4-year period starting in 2012 , a PA titre $\geqslant 1: 128$ was achieved in $\geqslant 80 \%$ of all age cohorts $>2$-years-old, with the following exceptions: the 12-year-old cohort in 2013 (79\%); the 14-year-old cohort in 2014-2015 (77-78\%), and the >70-year-old cohort in 2014 (77\%).

Between 2006 and 2015, the 3-year moving average of PA antibody titres $\geqslant 1: 16$ increased for most age 
(a) 2008

400

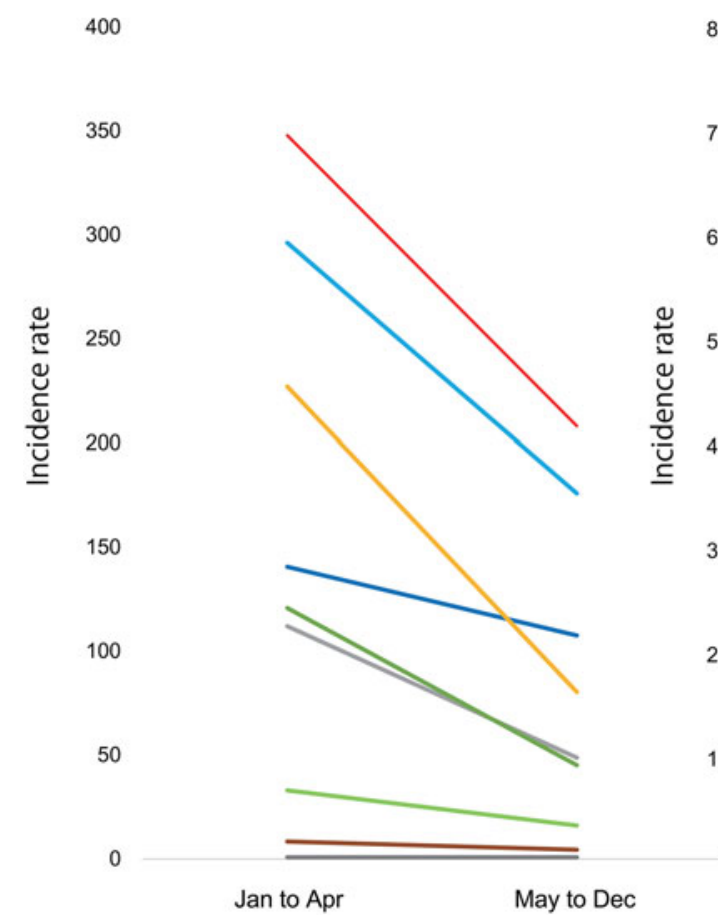

(b) 2009-2014
80

70

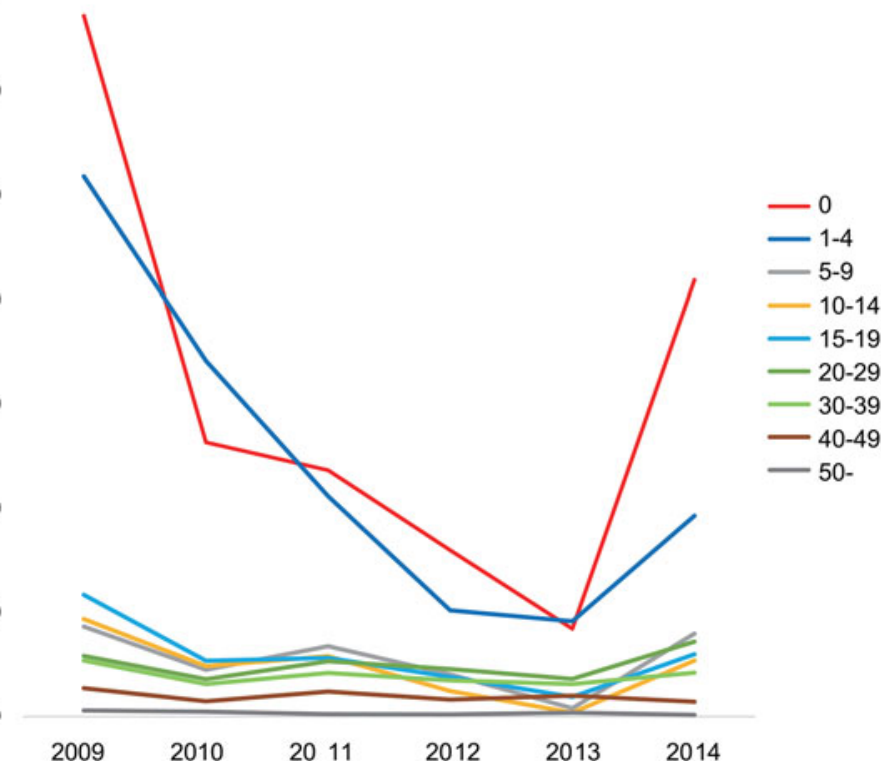

Fig. 3. Incidence rate of measles per million people by age cohorts. The incidence rate per million people was calculated using 2010 national census data, with the rates compared before and after the catch-up vaccination programme was implemented. The incidence rate for (a) 2008 and (b) 2009-2014 is shown. Again, 2015 was not included as only 36 cases were detected.

cohorts (by up to $+7 \%$ ), but with decreases for the 0 5 -month-old group (-8\% from $73 \%$ in 2006 ), 611-month-old group (-2\% from $14 \%$ in 2006), the 4-year-old group $(-1 \%)$ and about a half among the $30-39,40-49$ and $50-59$ age groups ( -1 to $-4 \%$ ). Similarly, the 3-year moving average of PA antibody titres $\geqslant 1: 128$ also increased overall, again with decreases among specific age groups: $0-5$ months and the $2-4,14,19$, and most of the 26-55- and the 60 -year-old groups $(-1$ to $-9 \%)$. Over the last 4 years, a relatively rapid decrease in PA antibody titres $\geqslant 1: 128$ were identified in the $0-5$-month-old group from $40 \%$ in 2012 to $18 \%$ in 2015 (Fig. 4).

\section{Epidemic virus and place of infection}

Virus genotyping was conducted using RT-PCR testing for $<30 \%$ of the cases between 2008 and 2010 and for approximately $60 \%$ of laboratory-confirmed cases between 2011 and 2014. In 2015, all 36 cases were genotyped. Within the typed virus samples $(n=$ 845 of the total 931 samples between 2010 and 2015), type D5, which has been the endemically

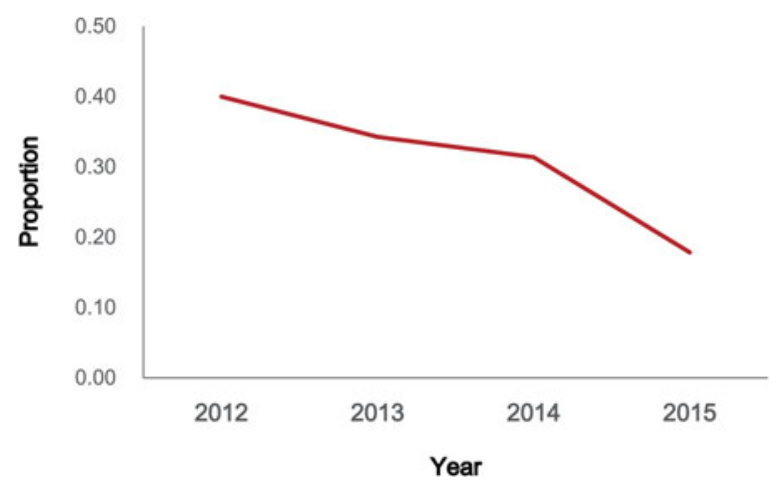

Fig. 4. Measles gelatin particle agglutination antibody titre $(\geqslant 1: 128)$ within the $0-5$-month age group for $2012-$ 2015 (a 3-year moving average). The data were obtained from the Infectious Agents Surveillance Report (IASR).

circulating strain in Japan, was only detected in 2010 (Fig. 5). Through 2011, the majority of cases were of the D4 genotype, which was associated with the measles virus imported to Japan. D9 was detected only in 2010 and 2011. The detection of D8 was highest in 2012 and 2015, and was the second highest genotype in 2013 and 2014. The B3 genotype was highest in 2013 and 2014. Genotype 'A', which was 


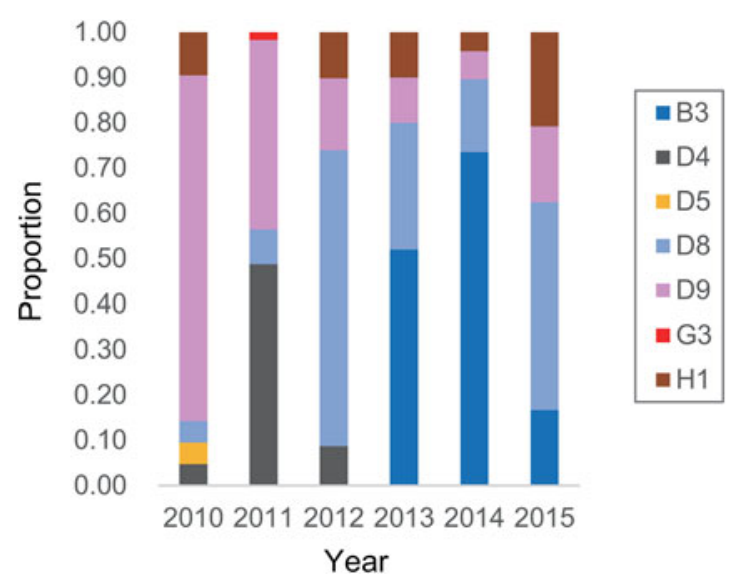

Fig. 5. The distribution of measles virus genotype. Numbers of each measles virus genotype detected via laboratory testing, as obtained from the Infectious Agents Surveillance Report (IASR) are shown.

confirmed as the vaccine strain, was detected in $4-31 \%$ cases throughout the period of observation. Genotype ' $A$ ' was not considered among cases of measles in the surveillance data and, therefore, was not included in Figure 5.

Of the countries in which the cases were suspected to have been infected ( $n=165$ available cases from 2008 to 2014), the majority of cases were infected in the Philippines $(50 \%)$, followed by China, Indonesia and Vietnam (each 7\%), Thailand (5\%) and Taiwan, Australia and France (each 2\%).

\section{DISCUSSION}

Our findings show that Japan's catch-up vaccination programme, which was implemented in 2008, enhanced population immunity by increasing the administration of a second vaccination dose. Furthermore, the increase in PA antibody titres $\geqslant 1: 16$ and $\geqslant 1: 128$ for the majority of sampled age cohorts $>2$-years-old after 2012 is indicative of an overall increase in measles immunity. As a result, a remarkable decrease in measles incidence was observed. In addition, no major peak of measles cases was identified after vaccination consolidation, suggesting that epidemic peaks may disappear after inhibition of massive and continuous virus transmission achieved by enhanced immunization [20-24]. Because measles epidemics occur periodically, even among cohorts with a relatively high vaccination coverage [1], observation and analysis of longer-term data are needed to confirm a lowered risk of outbreaks and longer time intervals between peaks. These data would provide more definitive evidence of the impact of the vaccination programme. The relatively higher incidence rate among age groups who had received either 'no' or 'one' dose of the vaccination indicates that one dose is insufficient for immunization, with two doses of the vaccination being required.

Although the incidence rate of measles declined among all age groups, higher incidence rate persisted in children 1-4-years-old. The most noteworthy finding was an increased incidence rate in 2014 for the 0 - and 1-4-year-old age groups. The reason is unknown, but the catch-up programme launched in 2008, which targeted children 13-16-years-old, may have been a contributing factor. This immunized group had a weaker natural immunity booster resulting from less exposure to large epidemics. Hence, it is possible that beginning in approximately 2014, 6 years after the catch-up vaccination initiative was implemented, more babies were born with less passive natural immunity [2, 25-28]. The women who received the catch-up vaccine were at an age to be giving birth around this time; a 16-year-old in 2008 would be 22 years of age in 2014, an age at which women in Japan are more likely to begin families. This hypothesis is supported by the lower passive immunity, as indicated by a PA antibody titre $\geqslant 1: 128$, in babies up to 5 months of age and, also, by a decreasing proportion of PA antibody titre $\geqslant 1: 128$ among individual in many of the older age cohorts over the last 4 years. Previous studies also concluded that there is likely a decay of induced immunity after several decades [2, 29]. These results indicate the potential need to consider implementing an immunity enhancement programme between periods of low vaccination coverage, with a natural booster and higher vaccination coverage, with lower levels of immunity booster.

The endemic Japanese virus genotype, D5, nearly disappeared after 2010, indicating that the recent epidemic was caused by imported viruses [30]. The highest frequency of importation of the measles virus was from the Philippines. The decrease in measles incidence in Japan after the end of 2014 coincided with the launch of the supplementary vaccination programme in the Philippines in September 2014 [31, 32]. At that time, the 1500 of cases recorded per month (June 2011September 2014) markedly decreased to 573 cases per month (October-December 2014), which appears to be associated with the decrease in measles virus importation into Japan at the end of 2014. Therefore, even with widespread vaccination coverage, which can be 
high enough to eliminate domestic endemicity, transmission via epidemic areas overseas may cause sporadic epidemics if immunity is incomplete. Among the age groups included in our surveillance data, the number of measles cases remained proportionally high among the 20-39 years cohort and the children 1-4-years-old across all years surveyed. The 20-39 age group might be more active and travel abroad more frequently, playing a major role in virus transmission from overseas. Because vaccination of individuals within the first year of life is considered to be less effective than vaccination provided at 12 months of age [2, 23] and also the immunisation for children may not be sufficient until the second-dose vaccination at 5-6-year-old [5], prevention of epidemics among infants should be enhanced by eliminating imported viruses from overseas as well as by containing endemic transmission through an increased immunization of older age groups. Therefore, monitoring and preventive measures should be undertaken, with a particular focus on individuals in the 20-39 years age group.

During periods in which the overall vaccination coverage increases, the epidemic is more likely to be associated with epidemic status overseas. During such a period, the domestic risk of measles cases can only be determined by monitoring the status of vaccination coverage and seroprevalence of measles antibody, not just the incidence rate as an increase in incidence is not immediately visible when an overseas epidemic declines. In other word, in such a period, measles surveillance needs to be active to test a sufficient number of individuals for seroprevalence of measles antibody and vaccination status, regardless of the size of detected outbreaks. Likewise, laboratory and RT-PCR testing of measles cases is especially important for confirmation of cases during a non-epidemic period when the public awareness for measles risk tends to be relatively low and detecting measles cases can be more difficult.

Although the incidence data used in this study could have included other cases with similar symptoms, the majority of the data was based on laboratory confirmation. However, data regarding vaccination status was limited to patient or parent self-reported information, which is a major limitation of our study with regard to examining the specific effect of an increase of vaccination coverage. In addition, because the vaccination coverage and the PA antibody surveillance for each age group was conducted in designated hospitals, these data may only represent the trends in that area served by each designated hospital. Similarly, because such active surveillance was conducted mainly for outpatients who visited the designated hospitals, those patients might have had more awareness of the importance and need for measles vaccination and, thus, a higher vaccination coverage than other cohorts who are less likely to visit hospitals. Also, differences in vaccination coverage among seroprevalence samples would influence the result of the ratio of the PA antibody. There are also other potential biases in how we conducted our data collection, including the selection and measurement of measles case and sampling of specimens for virus genotyping, which may have influenced our reliability in confirming the number of cases. The key to measles elimination is increasing vaccination coverage and monitoring of measles antibody status for all ages, as well as enhancing surveillance of both domestic and overseas incidences. Cross-country control strategies and activities will also be important for measles elimination.

\section{SUPPLEMENTARY MATERIAL}

The supplementary material for this article can be found at https://doi.org/10.1017/S0950268817001248.

\section{ACKNOWLEDGEMENTS}

The authors are grateful to Prof. Masao Mitsuyama of Kyoto University for his useful comments on the manuscript and to Dr. Hiroshi Yoshikura of the National Institute of Infectious Diseases for his critical comments on our study.

This study was supported by Kyoto University Startup Grant from Kyoto University.

The results mentioned here were presented, in part, to the Japanese Association of Infectious Diseases (Okayama, Western Japan) on 26 October 2014.

\section{REFERENCES}

1. Paunio M, et al. Explosive school-based measles outbreak: intense exposure may have resulted in high risk, even among revaccinees. American Journal of Epidemiology 1998; 148: 1103-1110.

2. Moss WJ, et al. Measles. Lancet 2012; 379: 153-164.

3. Choe YJ, et al. Evaluation of an expanded case definition for vaccine-modified measles in a school outbreak in South Korea in 2010. Japanese Journal of Infectious Diseases 2012; 65: 371-375.

4. World Health Organisation. Weekly epidemiological record 2010; vol. 85: 489-496 (http://www.who.int/wer/ 2010/wer8549.pdf). 
5. Yoshikura H. Relation between measles incidence and population size under the advanced vaccine program. Japanese Journal of Infectious Diseases 2012; 65: 88-91.

6. Gay NJ. The theory of measles elimination: implications for the design of elimination strategies. Journal of Infectious Diseases 2004; 189: S27-S35.

7. Regional Office for the Western Pacific, World Health Organization: Report of task force meeting on measles elimination in the Western pacific region, Manila, Philippines, 29-30 July 2004.

8. World Health Organisation. Brunei Darussalam, Cambodia, Japan verified as achieving measles elimination: Western Pacific Region achieves progress towards measles elimination, but challenges remain. 2015 (http://www.wpro.who.int/mediacentre/releases/ 2015/20150327/en/).

9. National Institute of Infectious Diseases. Infectious Agents Surveillance Report (http://www.nih.go.jp/niid/ en/).

10. Yoshikura H. Impact of population size on incidence of rubella and measles in comparison with that of other infectious diseases. Japanese Journal of Infectious Diseases 2014; 67: 447-457.

11. Ministry of Health, Labour, and Welfare. Deaths from measles by age (http://idsc.nih.go.jp/iasr/22/261/dj261a. html).

12. Progress towards eliminating measles in Japan, 2008. The weekly epidemiological record 2008; vol. 83: 351355.

13. Yoshikura H. Negative impacts of large population size and high population density on the progress of measles elimination. Japanese Journal of Infectious Diseases 2012; 65: 450-454.

14. Mossong $\mathbf{J}$, et al. Modeling the impact of subclinical measles transmission in vaccinated populations with waning immunity. American Journal of Epidemiology 1999; 150: 1238-1249.

15. Sodha SV, et al. Strengthening routine immunization systems to improve global vaccination coverage. British Medical Bulletin 2015; 113: 5-14.

16. Sheldon MR, et al. The use and interpretation of the Friedman test in the analysis of ordinal-scale data in repeated measures designs. Physiotherapy Research International 1996; 1: 221-228.

17. Taniguchi K, et al. Overview of infectious disease surveillance system in Japan, 1999-2005. Journal of Epidemiology 2007; 17: S3-S13.

18. Gu Y, et al. National surveillance of influenza-associated encephalopathy in Japan over six years, before and during the 2009-2010 influenza pandemic. PLOS ONE 2013; 8: e54786.

19. Statistics Bureau, Ministry of Internal Affairs and Communications. Population census (http://www.e-stat. go.jp/SG1/estat/eStatTopPortalE.do).

20. Fine PE, et al. Measles in England and Wales-II: the impact of the measles vaccination programme on the distribution of immunity in the population. International Journal of Epidemiology 1982; 11: 15-25.

21. Murdoch WW, et al. Spatial dynamics of measles epidemics. Trends in Ecology \& Evolution 2002; 17: 399-401.

22. Conlan AJ, et al. Seasonality and the persistence and invasion of measles. Proceedings of the Royal Society B: Biological Sciences 2007; 274: 1133-1141.

23. De Serres G, et al. Measles vaccine efficacy during an outbreak in a highly vaccinated population: incremental increase in protection with age at vaccination up to 18 months. Epidemiology \& Infection 1995; 115: 315-323.

24. Stone $\mathbf{L}$, et al. Seasonal dynamics of recurrent epidemics. Nature 2007; 446: 533-536.

25. Davidkin I, et al. Vaccine-induced measles virus antibodies after two doses of combined measles, mumps and rubella vaccine: a 12-year follow-up in two cohorts. Vaccine 1998; 16: 2052-2057.

26. Leuridan E, et al. Early waning of maternal measles antibodies in era of measles elimination: longitudinal study. British Medical Journal 2010; 340: c1626.

27. Pabst HF, et al. Reduced measles immunity in infants in a well-vaccinated population. Pediatric Infectious Disease Journal 1992; 11: 525-529.

28. Brugha $\mathbf{R}$, et al. A study of maternally derived measles antibody in infants born to naturally infected and vaccinated women. Epidemiology \& Infection 1996; 117: $519-524$.

29. Amanna IJ, et al. Duration of humoral immunity to common viral and vaccine antigens. New England Journal of Medicine 2007; 19: 1903-1915.

30. Takahashi T, et al. Ongoing increase in measles cases following importations, Japan, March 2014: times of challenge and opportunity. Western Pacific Surveillance and Response Journal 2014; 5: 31-33.

31. Takashima Y, et al. Progress toward measles elimination -Philippines, 1998-2014. Morbidity and Mortality Weekly Report 2015; 64: 357-362.

32. World Health Organization. Measles-rubella bulletin 2016; vol. 10 (http://iris.wpro.who.int/bitstream/handle/ 10665.1/12922/Measles-Rubella_Bulletin_2016_Vol_10_ No_02.pdf?ua=1). 